\title{
Distal Tubular Feedback in the Autoregulation of Single Nephron Glomerular Filtration Rate
}

\author{
L. Gabriel Navar, Thomas J. Burke, Roscoe R. Robinson, and \\ JAMES R. ClapP
}

From the Division of Nephrology, Department of Medicine, Duke University Medical Center, Durham, North Carolina 27710

\begin{abstract}
A B S T R A C T Renal clearance and recollection micropuncture experiments were conducted to evaluate the possible role of a distal tubular feedback mechanism in the phenomenon of renal autoregulation in dogs. Single nephron glomerular filtration rate (SNGFR) was measured from collection sites in both the proximal (proximal SNGFR) and distal tubules (distal SNGFR). Single nephron autoregulatory behavior was assessed by evaluating the response of $S N G F R$ to a reduction in renal arterial pressure imposed by means of an aortic constrictor. Whole kidney function was evaluated by parallel measurements of renal blood flow and inulin clearance. Whole kidney autoregulation was observed when renal arterial pressure was decreased from $141 \pm 3$ (SE) $\mathrm{mm} \mathrm{Hg}$ to $101 \pm 2 \mathrm{~mm} \mathrm{Hg}$; renal blood flow and GFR were not significantly altered from control values of $3.76 \pm 0.2 \mathrm{ml} / \mathrm{min} \cdot \mathrm{g}$ and $0.69 \pm 0.04 \mathrm{ml} / \mathrm{min} \cdot \mathrm{g}$ kidney weight, respectively. In 11 autoregulating preparations, proximal transit time was likewise unchanged from the control value of $26 \pm 2 \mathrm{~s}$, indirectly suggesting that the superficial nephrons also participated in the autoregulatory response. However, proximal SNGFR decreased significantly from $88 \pm 7 \mathrm{nl} / \mathrm{min}$ to $66 \pm 6 \mathrm{nl} / \mathrm{min}$, a re-
\end{abstract}

Portions of this study were presented in preliminary form at the Southern Section and national meetings of the American Federation for Clinical Research and the spring meeting of the American Physiological Society, 1973.

Dr. Navar's present address is the Department of Physiology and Biophysics, University of Mississippi Medical Center, Jackson, Mississippi 39216. Dr. Burke's present address is the Department of Physiology, University of Colorado, Denver, Colorado 80220.

This work was performed during Dr. Navar's tenure as a visiting scientist under the sponsorship of NIH Special Fellowship AM 52421. Dr. Burke was an NHLI postdoctoral trainee. Dr. Robinson is a Senior Investigator of the North Carolina Heart Association, and Dr. Clapp is an Investigator of the Howard Hughes Institute.

Received for publication 14 June 1973 and in revised form 13 September 1973. duction which was proportional to the decrease in arterial pressure. In 14 dogs in which both proximal SNGFR and distal SNGFR were measured at control

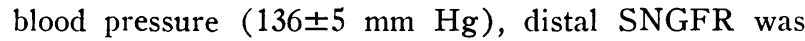
$47 \pm 4 \mathrm{nl} / \mathrm{min}$, a value significantly lower than that for proximal SNGFR $(79 \pm 6 \mathrm{nl} / \mathrm{min})$. In contrast to the results based upon proximal collections, distal SNGFR was not significantly altered following aortic constriction $(44 \pm 5 \mathrm{nl} / \mathrm{min}$ vs. $47 \pm 5 \mathrm{nl} / \mathrm{min})$ therefore exhibiting autoregulation in association with that observed for the whole kidney. These experiments indicate that though superficial nephrons do possess autoregulatory capability, interruption of distal delivery due to complete collection from the proximal tubule interferes with that nephron's ability to manifest an autoregulatory response. They support the concept that a feedback mechanism, related to some function of distal delivery, is of significance in the intrinsic regulation of SNGFR. The data further suggest that quantitative estimates of SNGFR based on complete proximal collections may not be representative of those throughout the superficial cortex of the dog, at least in certain experimental circumstances.

\section{INTRODUCTION}

The phenomenon of renal autoregulation is thought to reflect the activity of an intrinsic intrarenal mechanism which contributes to the control of renal hemodynamics $(1,2)$. This autoregulatory mechanism serves to adjust the intrarenal vascular resistance in such a way that renal blood flow $(\mathrm{RBF})^{\mathbf{1}}$ and glomerular filtration rate (GFR) are kept relatively constant (3) over a wide

\footnotetext{
${ }^{1}$ Abbreviations used in this paper: BP, blood pressure; $\mathrm{GFR}$, glomerular filtration rate; $\mathrm{PAH}, p$-aminohippurate; $\mathrm{RBF}$, renal blood flow; SNGFR, single nephron glomerular filtration rate; $\mathrm{TF} / \mathrm{P}$, tubular fluid to plasma inulin concentration.
} 
range of renal perfusion pressures (approximately 75$180 \mathrm{~mm} \mathrm{Hg}$ ). It may also contribute to the adjustments of renal vascular resistance that are known to occur in response to increased ureteral or plasma oncotic pressure $(4,5)$. Most lines of evidence have suggested that the requisite changes of renal vascular resistance are localized primarily to preglomerular sites, i.e., the afferent arteriole $(6-8)$. To explain this phenomenon, it has been proposed that afferent arteriolar resistance may be modulated via the influence of a feedback control system involving an interplay of signals between the macula densa region of the distal convoluted tubule and the afferent arteriole $(2,4,5,9,10)$. For example, decreased renal perfusion pressure and an associated reduction of RBF and GFR may effect a diminished delivery of tubular fluid to the distal nephron. One or more functions of diminished distal delivery may be sensed by the cells of the macula densa which then, in turn, contribute to the generation and transmission of signals that effect dilation of the afferent arteriole and the restoration of GFR and $R B F$ toward their initial values. Conversely, one or more functions of increased distal delivery might serve to bring about vasoconstriction of the afferent arteriole and decreased $R B F$ and $G F R$ via the same pathway.

At the single nephron level, renal micropuncture techniques have been used to examine the validity of the macula densa-afferent arteriole feedback hypothesis, primarily in the rat. It was first reported that the retrograde injection of a sodium-containing fluid into the lumen of the distal tubule may be accompanied by proximal luminal collapse (10). Later, it was reported that the single nephron glomerular filtration rate (SNGFR), as measured from complete collections of proximal tubular fluid and thus under conditions of diminished distal delivery due to the interposition of an intraluminal oil block between the proximal site of sampling and the distal tubule, is higher than SNGFR as measured from collections of distal tubular fluid (11). Also, SNGFR (from proximal collections) was reported to be related inversely to the rate of distal perfusion (via puncture sites in the late proximal tubule) with modified Ringer's solution (12). Similarly, when changes of glomerular hydrostatic pressure were assessed indirectly by measurements of proximal intraluminal pressure during the stopped-flow of tubular fluid, it was suggested that glomerular hydrostatic pressure is responsive to changes of distal perfusion via late proximal puncture sites $(13,14)$. These results have been cited in support of the concept that SNGFR is regulated, at least in part, by some function of volume delivery to the distal nephron. However, in striking contrast, other investigators have been unable to confirm the validity of these relationships in the rat, i.e., differing rates of distal perfusion have not been accompanied by changes of SNGFR (15) and measurements of SNGFR have been found to be similar in the same nephron, irrespective of the site of sampling (proximal versus distal tubule) (16). In addition, it has been reported that direct measurements of glomerular hydrostatic pressure in a mutant strain of Wistar rats are unresponsive to the induction of complete proximal luminal blockade (17). Thus, the available evidence in support of the presence of a distal tubular feedback system in the rat is conflicting and inconclusive.

Mechanistically, it is logical to assume that the activity of a possible distal tubule-afferent arteriole feedback system and the phenomenon of renal autoregulation are interrelated. Thus far, however, the relationship between the responsiveness of such a feedback system in single nephrons, if present, and that of whole kidney autoregulation has not been established. To the extent that whole kidney autoregulation reflects the collective responsiveness of single nephrons, it would seem important to establish the integrity of whole kidney autoregulation in any study of the possible contribution of a distal tubule feedback system to the autoregulatory behavior of single nephrons. Unfortunately, the integrity and/or character of whole kidney responsiveness cannot be ascertained with reliability from the results of previous renal micropuncture studies in the rat. For this reason, the present micropuncture studies were initiated in the dog under circumstances in which overall renal autoregulatory ability was known to be intact and its relationship to single nephron behavior could be established. Studies were carried out in the dog because of the relative ease with which autoregulatory behavior can be assessed at differing degrees of renal perfusion pressure and the wealth of available data in this species against which our own results could be compared.

SNGFR and whole kidney filtration rate and renal blood flow were measured at differing levels of mean arterial pressure within the autoregulatory range; measurements of SNGFR were based on complete collections of tubular fluid from both proximal and distal sites of collection. Since such measurements require the interposition of an intraluminal oil block and the consequent interruption of delivery to those sites distal to the point of sampling, collections from proximal tubules resulted in the cessation of normal delivery to the distal nephron whereas collections from the distal tubule did not interfere, at least greatly, with the normal delivery of tubular fluid to the region of the macula densa. Thus, a comparison between measured values for SNGFR from the two collection sites provided direct evidence on the possible role of changing distal delivery in the autoregulation of SNGFR, as well as an assessment of single nephron responsiveness to changing renal perfusion pressure. 


\section{METHODS}

Studies were carried out in 34 mongrel dogs of both sexes (body wt: 16-25 kg). Anesthesia was induced by the i.v. administration of sodium pentobarbital $(30 \mathrm{mg} / \mathrm{kg})$ and supplemental doses were administered as necessary throughout the experiment. A cuffed endotracheal tube was introduced and the animals were allowed to ventilate spontaneously on room air. Both carotid arteries were isolated and a polyethylene catheter was inserted into one for continuous measurements of arterial blood pressure via an attached pressure transducer. Whenever necessary, the remaining carotid artery was partially constricted in order to maintain the control blood pressure within the range of 130 $150 \mathrm{~mm} \mathrm{Hg}$. Two peripheral foreleg veins were catheterized; one of these catheters was used to infuse a solution of inulin and $p$-aminohippurate $(\mathrm{PAH})$ at a rate sufficient to achieve a plasma concentration approximating 75 and 1 $\mathrm{mg} / 100 \mathrm{ml}$, respectively. A catheter was inserted through one femoral artery into the aorta to a level slightly below the left renal artery and then connected to a Statham pressure transducer (Statham Lab., Inc., Hato Rey, Puerto Rico). This catheter was used for estimates of renal arterial pressure and the collection of arterial blood samples. Another catheter was inserted through the contralateral femoral artery so that its tip was placed just above the level of the renal arteries. This catheter was used for the injection of $1-2 \mathrm{ml}$ volumes of a $10 \%$ solution of lissamine green dye to facilitate the identification of late proximal and distal tubules and to determine the transient time of dye through the superficial nephrons. The left kidney, renal artery and aorta were exposed via a left flank incision. An adjustable brass clamp was placed around the aorta approximately $1 \mathrm{~cm}$ above the left renal artery. An electromagnetic flow transducer (Carolina Medical Electronics, Inc., King, N. C.) was then placed around the isolated renal artery and the left ureter was catheterized. To facilitate subsequent clearance measurements, urine flow was increased modestly by an i.v. infusion of $100-200 \mathrm{ml}$ of isotonic saline solution over a $10-20$-min period. The left kidney was then exposed and mounted on a lucite holder and the kidney surface was prepared for micropuncture as previously described (18). After the kidney had been mounted and prepared for micropuncture, its autoregulatory ability was assessed by briefly clamping the renal artery and noting the temporary but typical hyperemic phase of the vascular recovery pattern following the release of constriction (19). Renal blood flow and blood pressure were recorded continuously on a Sanborn recorder (Hewlett-Packard Co., Palo Alto, Calif.) via appropriate catheters in the femoral and carotid arteries.

In preparation for micropuncture, a small area $\left(2 \mathrm{~cm}^{2}\right)$ of the renal capsule was removed and the surface of the kidney was bathed continuously with mineral oil. After an injection of lissamine green dye, proximal transit times were measured as the interval between the first surface blush of green dye and its disappearance from the last surface convolution of proximal segments. Distal tubules were identified by the reappearance of green dye in sparsely distributed tubular segments $20-40$ s after the dye had disappeared from proximal segments. Late proximal segments and, when observed, distal tubular segments were then identified and punctured with a small-tipped (1-2 $\mu \mathrm{m})$ pipette containing nigrosin dye. A small amount of dye was injected into the lumen to determine the direction of tubular fluid flow, and the tubule was marked for recollec- tion by a tiny intraepithelial deposition of dye as the pipette was withdrawn.

Measurements of SNGFR were derived from timed and complete collections of tubular fluid according to the following technique. A droplet of Sudan black-stained siliconolive oil mixture $(3: 1)$ was first injected into the tubule lumen and the direction of fluid flow, as assessed earlier by an injection of nigrosin dye, was confirmed. A column of oil sufficient to fill the tubular lumen to a distance of approximately five tubular diameters was injected. A slight negative pressure was applied to initiate the collection which then proceeded without continuous aspiration. The delay in time between the injection of the oil column and the initiation of sampling was kept at a minimum; overall, all collection times were at least 20 times greater than the period of stopped fluid flow along the tubule $(20$, $21)$. Intermittant negative pressure was used when necessary to maintain the oil column in a constant distal position throughout the entire collection period. Proximal collections were usually of $1 \mathrm{~min}$ duration, at which time a volume of tubular fluid sufficient for microanalysis had been obtained. Collection times from distal tubules were longer, always being at least $2 \mathrm{~min}$ in duration and sometimes lasting as long as $5 \mathrm{~min}$. Care was taken to insure that the directional flow of distal fluid during collection was identical to that determined previously and, in most collections, the pipette was inserted against the direction of flow. Special care was taken to insure that the collection pipette was well threaded into the distal lumen and, for the most part, the criteria outlined by Wright and Giebisch were fulfilled (21). After the completion of collection, the pipette was removed from the tubule lumen and a small volume of mineral oil on the surface of the kidney was aspirated into the tip of the collection pipette. When both proximal and distal tubular fluid collections were being taken, all distal collections were done first or alternated with proximal collections. Collections were not initiated for at least $1 \mathrm{~h}$ after the saline infusion and $30 \mathrm{~min}$ after any lissamine green injections.

The usual experimental protocol was as follows. After the administration of priming doses of inulin and $\mathrm{PAH}$ and an equilibration period of $45-60 \mathrm{~min}$ duration, two to three timed collections of urine were obtained sequentially at control levels of blood pressure in association with simultaneous collections of tubule fluid. Arterial blood samples were usually collected after every second collection of tubular fluid. The aortic constrictor was then tightened slowly to decrease renal arterial pressure to a level ranging between 90 and $110 \mathrm{~mm} \mathrm{Hg}$. Continuous monitoring of renal blood flow permitted an immediate assessment of autoregulatory efficiency. At this lower blood pressure, urine flow was still sufficiently adequate to permit valid clearance determinations without undue delay; one or two timed urine collections were obtained at the lower level of renal perfusion pressure. Whenever possible, recollections of tubular fluid were obtained from the same puncture sites that had been used previously at higher levels of blood pressure. When measured, three to four estimates of proximal transit time were obtained at each level of blood pressure; similarly, whenever possible, recollections of tubule fluid for measurements of SNGFR were obtained from three to six proximal or distal puncture sites at each level of renal perfusion pressure. The experimental sequence was reversed in six animals, i.e., clearance measurements and micropuncture were first carried out at lower levels of 
blood pressure during the presence of partial aortic constriction.

Measurements of whole kidney renal blood flow and GFR were carried out at two levels of renal perfusion pressure in 28 of the 34 animals; in six dogs, measurements were obtained at higher blood pressures alone. Parallel measurements of SNGFR were obtained from proximal and distal collection sites in each of the latter six animals in which GFR and RBF were assessed only at higher blood pressures. Recollection measurements of SNGFR from proximal and/or distal sites were obtained in 23 of the 28 animals in which RBF and GFR were measured at two levels of renal perfusion pressure (the response of proximal transit time alone was assessed in the remaining five dogs within this group). Whenever assessed, whole kidney autoregulation was said to be "highly efficient" when a $20-30 \%$ reduction of renal perfusion pressure was accompanied by the maintenance of renal blood flow at values within $10 \%$ of those observed at higher blood pressures.

The volume of tubular fluid collections was measured with a calibrated micropipette using a slide comparator (Gaertner Scientific Corp., Chicago, Ill.) and tubular fluid inulin concentration was measured in triplicate using a microfluorometric method (22). Inulin in plasma and urine was measured using an anthrone colorometric technique (23). Urine and plasma PAH concentrations were determined using a standard colorometric technique (24). Micro-hematocrit measurements were performed on all arterial blood samples. Plasma protein concentrations were determined by light refractometry (American Optical Corp., Scientific Instruments Div., Buffalo, N. Y.). GFR and $\mathrm{PAH}$ clearances were calculated by the standard clearance formula. SNGFR was calculated as the product of the volume flow of tubular fluid $(\mathrm{nl} / \mathrm{min})$ and the ratio of the tubular fluid to plasma inulin concentration (TF/P). Sodium concentrations in plasma and urine were determined by flame photometry.

At the termination of each experiment, the electromagnetic blood flow transducer was calibrated directly in situ by catheterizing the renal artery and obtaining a timed collection of blood. The kidney was then excised, stripped of fat and surrounding tissue, blotted dry, and weighed. Direct measurements of renal blood flow correlated well with values derived from measurements of $\mathrm{PAH}$ clearance and they were used preferentially in most experiments. Both GFR and RBF were expressed in terms of $\mathrm{ml} / \mathrm{min}$ per gram of kidney weight $(\mathrm{ml} / \mathrm{min} \cdot \mathrm{g})$. Standard statistical tests were applied to the data and the paired $t$ test was used to assess the statistical significance of differences (25).

\section{RESULTS}

Hemodynamic response to aortic constriction. Judgements on the ability of the kidney to autoregulate RBF in response to a decrease in blood pressure were usually based on measures of RBF as obtained with the use of the electromagnetic flowmeter. A change in RBF of less than $10 \%$ in response to a $25-30 \%$ reduction of blood pressure was considered to reflect the pressure of high autoregulatory efficiency. Overall, these strict criteria were satisfied fully in 23 of 28 dogs $(82 \%)$ in which whole kidney autoregulatory efficiency was assessed. Recollection renal micropuncture experiments were carried out in 18 of the 23 animals exhibiting a high degree of autoregulatory efficiency (proximal transit times alone were assessed in the remaining five dogs). Table I portrays the average values for several indices of renal function from the 18 recollection micropuncture experiments. A decrease in mean arterial pressure from an average of $141-101 \mathrm{~mm} \mathrm{Hg}$ was accompanied by an appropriate autoregulatory adjustment of intrarenal vascular resistance; renal blood flow was essentially unchanged and GFR remained within $5 \%$ of the average value at higher blood pressures.

The individual results for $\mathrm{RBF}, \mathrm{GFR}$, and proximal transit time from each of the 18 micropuncture studies and 11 transit time studies are shown in Fig. 1. In a manner similar to RBF and GFR, the average proximal transit time was not altered significantly $(26 \pm 2 \mathrm{~s}$ vs. $27 \pm 2 \mathrm{~s}$ ) thus suggesting that the entire population of superficial nephrons may have participated in the autoregulatory response.

Despite the maintenance of $R B F$ and GFR in response to a reduction of arterial blood pressure, urine flow and

TABLE I

Hemodynamic and Renal Function Data at Control and Reduced Blood Pressure*

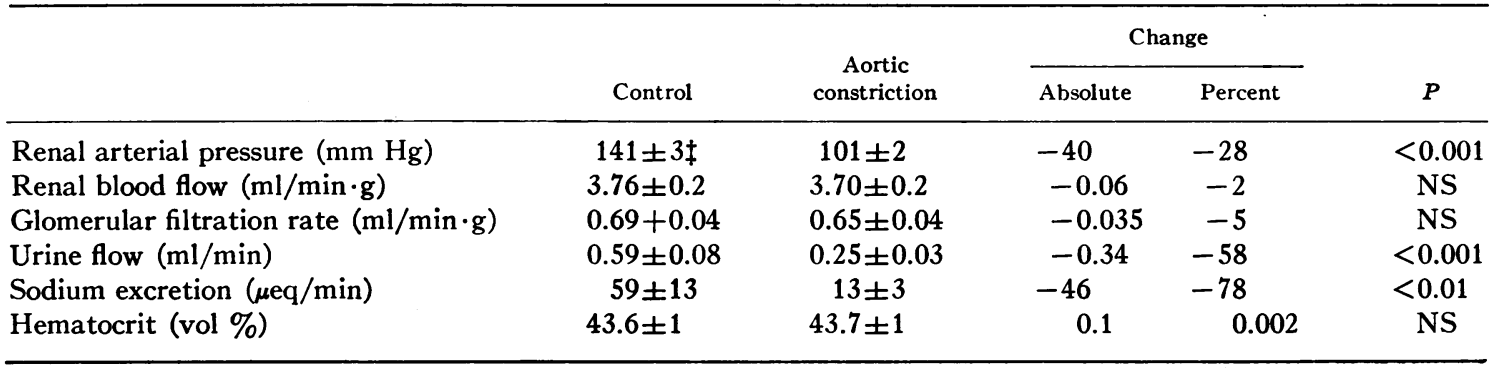

Kidney weight $=45.4 \pm 2.4 \mathrm{~g}$; Plasma protein concentration $=6.01 \pm 0.24 \mathrm{~g} / 100 \mathrm{ml}$.

Plasma sodium concentration $=146 \pm 1.3 \mathrm{meq} /$ liter.

* Data from 18 micropuncture experiments demonstrating high autoregulatory efficiency.

$\ddagger$ Mean $\pm \mathrm{SE}$. Where appropriate data refers to single kidney alone. 

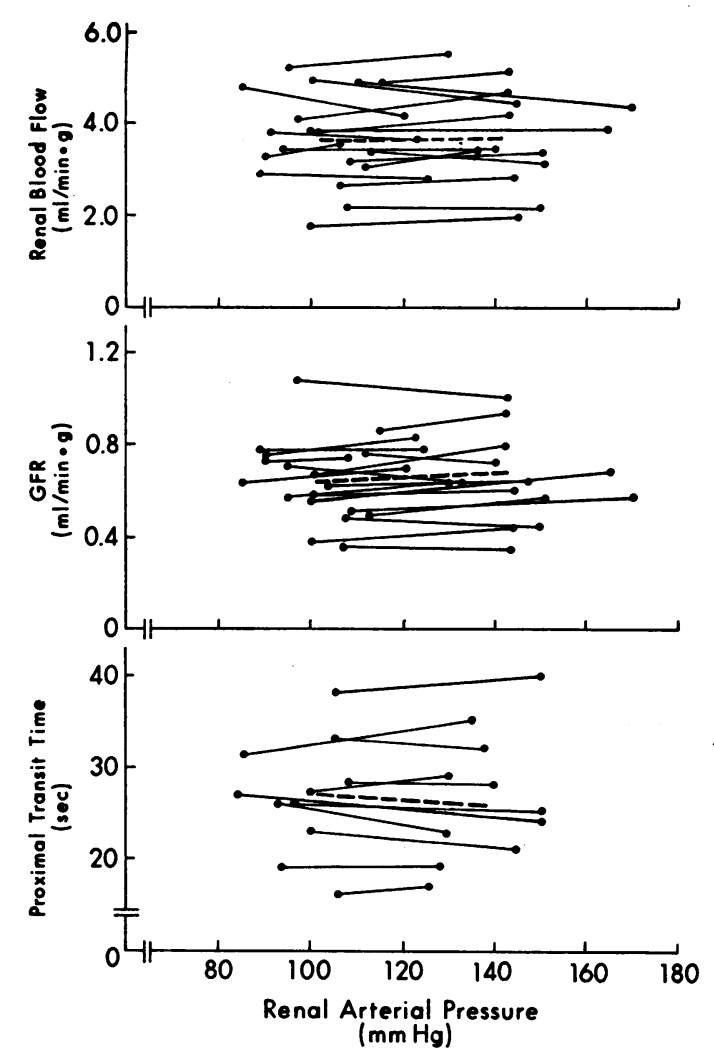

Figure 1 Response of renal blood flow (top panel), GFR (center panel), and proximal transit time (lower panel) to changes in renal arterial pressure due to aortic constriction. Individual experiments are plotted as solid lines while dashed lines designate mean results.

sodium excretion decreased consistently and significantly (Table I); urine flow fell by $58 \%$ and sodium excretion was reduced by $78 \%$. The observed reduction of water and electrolyte excretion in response to changes in arterial pressure even in the absence of apparent changes in RBF and/or GFR can be ascribed presumably to the phenomenon of "pressure diuresis," an event whose mechanism is not yet understood completely $(3,26)$. Its appearance in these experiments was taken as further evidence that the responsiveness of kidneys prepared for micropuncture was comparable to that observed in other experimental settings.

In the five dogs in which autoregulatory efficiency was less complete, average RBF fell by $29 \%$ after a decrease in blood pressure (BP) of $32 \%$, and whole kidney GFR fell by $18 \%$.

Response of single nephron filtration rate to aortic constriction. Technically satisfactory collections of proximal fluid were obtained at both levels of arterial pressure in each of the 18 "high efficiency" preparations and in each of the five "low efficiency" preparations. Measurements of SNGFR based on proximal collections (proximal SNGFR) were compared at the two arterial pressures; the results are shown in Fig. 2 where each point represents the average value for 3-6 recollection measurements in a single animal. Even in those animals exhibiting "high" autoregulatory efficiency (solid circles), aortic constriction was attended by a consistent and significant reduction of proximal SNGFR; overall, SNGFR decreased measurably in 39 of the 50 individual tubules in which recollections were obtained.

In the 18 high efficiency dogs, volume flow of proximal fluid at higher levels of $\mathrm{BP}$ averaged $60 \pm(\mathrm{SE}) 6 \mathrm{nl} / \mathrm{min}$, decreasing to $46 \pm 5 \mathrm{nl} / \mathrm{min}$ in the presence of aortic constriction. On the average, such a decrease was noted in 15 of the high efficiency animals $(P<0.01)$. It was this decrease in the volume flow of proximal fluid that contributed most of the observed reduction of SNGFR since the proximal $\mathrm{TF} / \mathrm{P}$ inulin ratio did not change significantly $(P<0.1)$, averaging $1.46 \pm 0.05$ at the higher pressure and $1.48 \pm 0.06$ at the reduced pressure. It is of interest to note that in these efficiently autoregulating dogs, decreases in arterial pressure did not disrupt proximal glomerular-tubular balance. Overall, the average recollection SNGFR from these 18 dogs decreased from $88 \pm 7$ to $66 \pm 6 \mathrm{nl} / \mathrm{min}$. The percentage decrease in SNGFR $(-25 \%)$ resembled that exhibited by the reduction in arterial pressure $(-28 \%)$.

Fig. 2 also contains the results from the five studies in which RBF was autoregulated poorly (open circles).

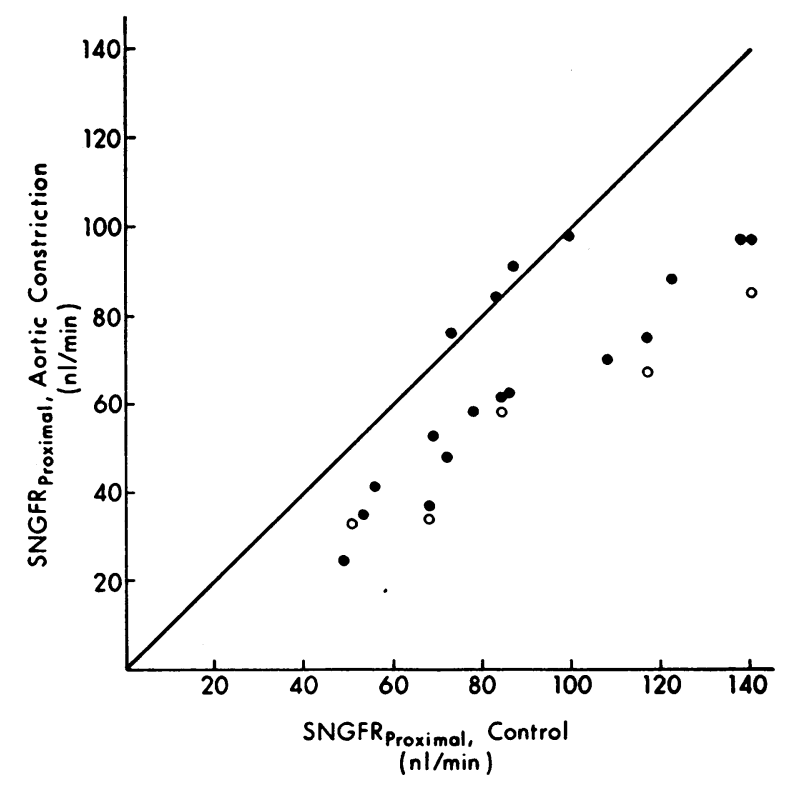

Figure 2 Effect of a reduction in blood pressure on SNGFR as measured from proximal tubular collections. Closed circles ( $\bullet$ ) represent data obtained from dogs exhibiting high degree of autoregulatory efficiency. Open circles $(\mathrm{O})$ are from dogs showing incomplete autoregulatory efficiency. 
SNGFR decreased by $40 \%$ in these five dogs $(92 \pm 12-$ $55 \pm 7 \mathrm{nl} / \mathrm{min}$ ) falling to a somewhat greater degree than that observed in high efficiency dogs. In these experiments, the TF/P inulin ratio increased slightly from 1.57 to 1.7 .

In 6 of 18 high efficiency experiments, recollections were made after the release of aortic constriction and the return of blood pressure to higher values. In these dogs, the release of aortic constriction was accompanied by a parallel restoration of proximal SNGFR to higher values (73-89 $\mathrm{nl} / \mathrm{min})$. The increase of SNGFR was significant $(P<0.05)$, thus demonstrating that the response of proximal SNGFR to changes in BP was reversible.

In 8 of the 18 high efficiency experiments and in 6 additional experiments in which aortic constriction was not employed, proximal and distal tubular collections were obtained from the same kidney in order that the two values for SNGFR (as derived from the two collection sites) could be compared at higher blood pressures alone. The individual values from each dog were averaged and they are presented in Fig. 3. In every instance, the average distal SNGFR was lower than the average proximal SNGFR; the proximal SNGFR averaged $79 \pm 6 \mathrm{nl} / \mathrm{min}$ whereas the distal SNGFR averaged $47 \pm 4 \mathrm{nl} / \mathrm{min}$. There were, of course, clear differences in the $\mathrm{TF} / \mathrm{P}$ inulin ratios from the two collection sites, those from the proximal collections averaging $1.57 \pm 0.09$ while those from the distal tubule averaged $3.78 \pm 0.21$. Volume flow from the proximal tubule averaged $51 \pm 3$

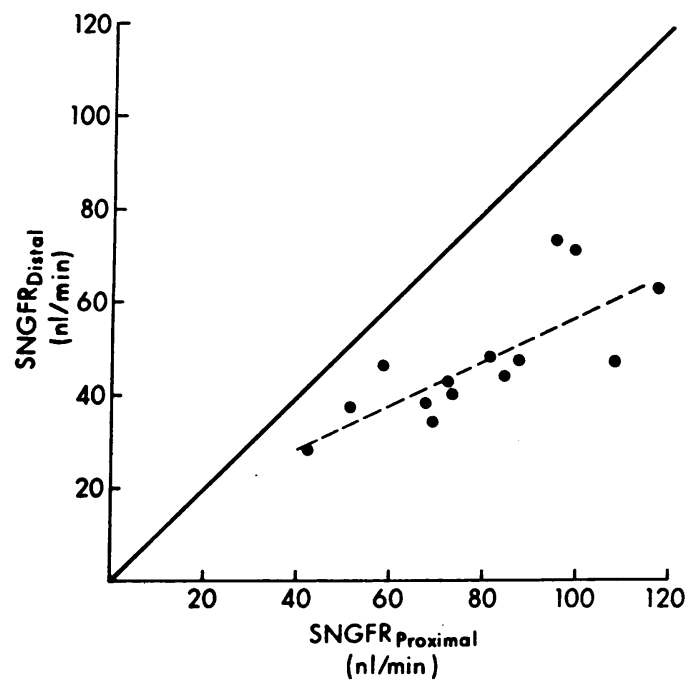

FIGURE 3 Comparison of SNGFR values obtained from proximal and distal tubular collections. Each point represents the mean data from one experiment. Regression equation is plotted as dashed line and is $y=x(0.48)+8.7$. The slope of the regression equation is significantly different from the slope of the identity line. In this series, arterial pressure was $136 \pm 5 \mathrm{~mm} \mathrm{Hg}, \mathrm{RBF}$ was $3.64 \pm 0.28$ $\mathrm{ml} / \mathrm{min} \cdot \mathrm{g}$ and $\mathrm{GFR}$ was $0.66 \pm 0.03 \mathrm{ml} / \mathrm{min} \cdot \mathrm{g}$.

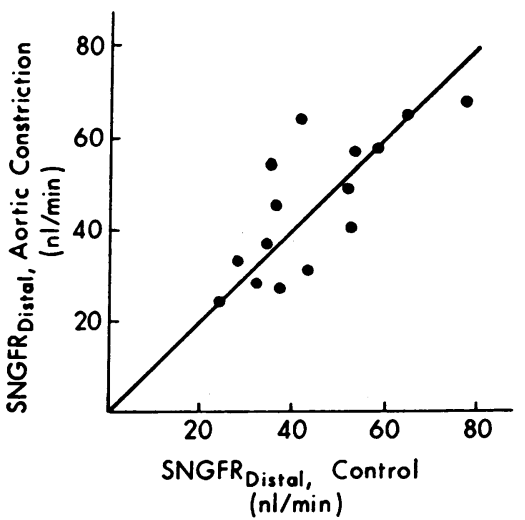

FIGURE 4 Comparison of SNGFR values obtained from distal tubule punctures at control and reduced arterial pressure. Individual data points from 15 tubules in eight dogs are plotted.

$\mathrm{nl} / \mathrm{min}$ while that from distal collections averaged $13 \pm$ $1 \mathrm{nl} / \mathrm{min}$.

In contrast to the clear decrease in proximal SNGFR during reduced arterial pressure, distal SNGFR was not significantly altered following $z$ reduction of mean arterial pressure. The results of distal recollections from 15 tubules in eight of eighteen "high efficiency" dogs are shown in Fig. 4 for the two levels of blood pressure. Distal SNGFR from these 15 tubules averaged $44 \pm 5 \mathrm{nl} / \mathrm{min}$ at higher blood pressures $(140 \pm 5 \mathrm{~mm} \mathrm{Hg})$ and $47 \pm 5 \mathrm{nl} / \mathrm{min}$ at lower blood pressures $(102 \pm 3 \mathrm{~mm}$ $\mathrm{Hg}$ ). Thus, autoregulation of superficial SNGFR was apparent when measurements of SNGFR was based on

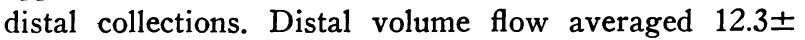
$0.7 \mathrm{nl} / \mathrm{min}$ at higher blood pressures and $10.5 \pm 1.5 \mathrm{nl} /$ $\mathrm{min}$ at reduced arterial pressure. This reduction of tubular volume flow was attended by a parallel increase of the $\mathrm{TF} / \mathrm{P}$ inulin ratio following aortic constriction (3.64 $\pm 0.3-4.58 \pm 0.6)$.

Of the five poorly regulating dogs in which proximal collections were made at both control and reduced pressure, distal collections were accomplished in only one and only at control blood pressure. Distal tubular data were obtained in two other poorly regulating experiments which were not carried to completion. At an average control blood pressure of $143 \mathrm{~mm} \mathrm{Hg}$, the average difference between proximal SNGFR and distal SNGFR was $13 \mathrm{nl} / \mathrm{min}$, substantially less than the differences observed in high efficiency autoregulatory dogs. Distal recollections following aortic constriction were attempted in these dogs and could not be satisfactorily accomplished. It appeared that distal flow rate had decreased markedly and sufficient volume for analysis could not be obtained.

Relationships between SNGFR and renal arterial pressure. Fig. 5 shows the relationships between renal 


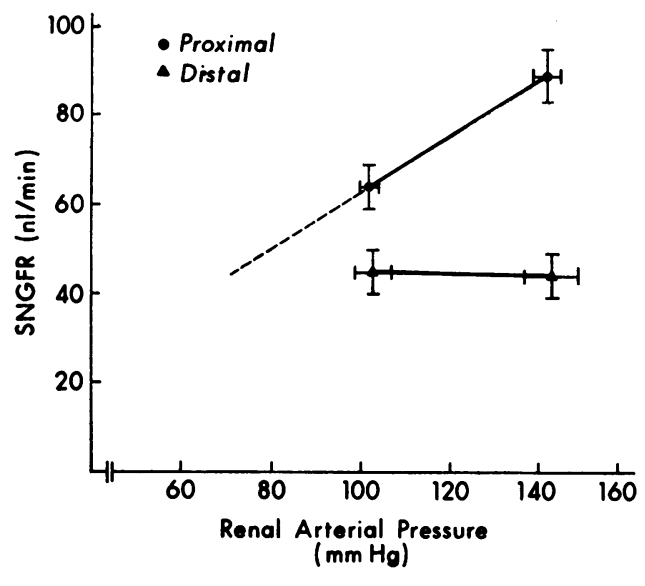

Figure 5 Relationships between renal arterial pressure and SNGFR as measured from proximal tubule and distal tubule collections. Circles $(\bullet)$ represent mean data obtained from proximal tubule collections. Triangles $(\boldsymbol{\Delta})$ represent mean data obtained from distal tubule collections. Only data from the experiments demonstrating "high autoregulatory" efficiency were used for this graph. Dashed line extends the proximal relationship to level of distal values.

arterial pressure and SNGFR as measured from either proximal or distal collection sites. At the higher arterial pressure, proximal and distal values for SNGFR differed markedly. However, at lower arterial pressures, the difference was less apparent since distal measurements of SNGFR remained unchanged while those for proximal SNGFR decreased measurably, almost in direct proportion to the decrease in arterial pressure. The dashed line (Fig. 5) extrapolates the measured relationship between proximal and distal SNGFR to that which might obtain at arterial pressures of $80-90 \mathrm{~mm} \mathrm{Hg}$; it pre-

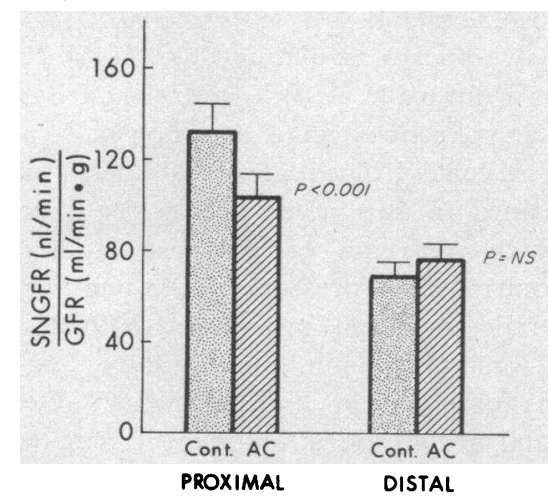

Figure 6 Effect of aortic constriction $(A C)$ on the ratio of nephron filtration rate to whole kidney glomerular filtration rate. Results from both the proximal and distal tubular collections are presented at control pressures (Cont.) and following reduction of blood pressure $(A C)$. Results from all experiments were used for this analysis. dicts that measurable differences between proximal SNGFR and blood pressure would not obtain as arterial blood pressure is reduced to the lower limits of the autoregulatory range.

Relationship between $S N G F R$ and kidney GFR. To determine the degree of association between the changes in SNGFR and those for whole kidney GFR, the ratio SNGFR: GFR was calculated utilizing measurements of SNGFR from both proximal and distal collections at the two levels of arterial blood pressure. When the data for proximal SNGFR are utilized in this manner (Fig. 6), a clear-cut dissociation is observed between the single nephron responsiveness and that in the whole kidney. This dissociation, as outlined previously, is almost entirely due to the fact that an appropriate autoregulatory response was observed at the whole kidney level, but not when SNGFR was measured by means of proximal collections. In contrast, when data for distal measurements of SNGFR are used (Fig. 6), it can be seen that the ratio $S N G F R$ : GFR remains essentially unchanged in response to a reduction in arterial pressure. Thus, when based on values for distal SNGFR, a close and appropriate association was maintained between the response of superficial nephrons and that for the total nephron population following reductions in renal arterial pressure from 140 to $102 \mathrm{~mm} \mathrm{Hg}$.

\section{DISCUSSION}

Previous micropuncture studies of single nephron responsiveness to alterations in arterial pressure have rarely been carried out under circumstances in which renal autoregulation was known to be intact. In one such attempt in seven dogs, Liebau, Levine, and Thurau (27) were unable to apply an aortic clamp successfully and therefore resorted to the use of manipulations that also effected an alteration of total systemic arterial pressure, i.e., carotid sinus denervation and pharmacologically induced hypotension. While it was stated that SNGFR (derived from collections of proximal fluid) was related linearly to whole kidney GFR and that both indices of filtration rate exhibited autoregulation in response to changes of blood pressure, the authors did not present specific data on measurements of changes in SNGFR in the dogs in which renal arterial pressure was altered directly. Because of our own uncertainty regarding the efficiency of whole kidney autoregulation in dogs prepared for renal micropuncture, its documentation was felt to comprise an essential part of this study of single nephron responsiveness to changes of renal arterial pressure. The present demonstration that whole kidney autoregulation is intact, at least in many dogs prepared for micropuncture, provided evidence of an acceptable environment in which the autoregulation of superficial SNGFR could be evaluated. Furthermore, 
the constancy of proximal transit time at differing levels of arterial pressure in those animals exhibiting a high degree of whole kidney autoregulatory efficiency suggested that whole organ responsiveness was shared by the entire population of superficial nephrons.

The present observations demonstrate that a reduction of blood pressure within the autoregulatory range (and the parallel maintenance of whole kidney GFR) is associated with a reduction of superficial SNGFR when the latter index of filtration rate is based on proximal collections and distal delivery has been interrupted. The decrease in proximal SNGFR was due solely to the decrease in volume flow and fractional reabsorption was not altered in the autoregulating preparations. This is in contrast to reports in which aortic constriction sufficient to decrease whole kidney renal plasma flow and GFR resulted in an increased proximal fractional reabsorption $(28,29)$. Consequently, it would appear that proximal glomerular-tubular balance is maintained more efficiently following aortic constriction in well autoregulating preparations. On the average, the relative decrease of proximal SNGFR was almost proportional to the relative decrease in blood pressure, thereby suggesting that proximal collections for measurements of SNGFR are accompanied by an inhibition of autoregulatory responsiveness in the individual nephron. This absence of autoregulation within the individual nephron leads to the observed dissociation between the response of proximal SNGFR and whole kidney GFR (Fig. 6). In contrast to the results derived from proximal collections, measurements of SNGFR via distal collections (distal SNGFR) indicated that SNGFR was not altered significantly following blood pressure reduction, thus suggesting that whole kidney autoregulation was, in fact, accompanied by a parallel degree of autoregulation at the single nephron level when distal delivery was presumably well maintained. In brief, these findings provide further evidence in support of the hypothesis that a feedback loop exists between some portion of the distal nephron and the arteriolar vasculature of its glomerulus. Although firm statements cannot be made, it is reasonable to assume that an important segment of the feedback loop may be localized to the macula densa.

It must be emphasized that the results of these studies do not bear on the specific nature of the feedback mediator. Perhaps it can be assumed that one or more factors associated with changing volume delivery to the distal tubule may serve in that capacity. While it has been suggested that some function of total solute delivery $(9,19)$ or sodium load $(2,10,12)$ is important to the feedback stimulus and that the renin-angiotensin system may be involved as the effector agent $(10,30)$, the actual validity of these suggestions remains uncertain.
At present, the exact nature of the specific mediator of a regulatory feedback response is unknown.

The present demonstration of a difference between measured values for proximal and distal SNGFR is in agreement with earlier findings in rats $(11,20)$. The higher proximal SNGFR suggests that interruption of normal volume delivery to the distal tubule alters the feedback stimulus and thereby leads to an elevation of SNGFR in that nephron. One means by which an increased SNGFR might occur would involve the appearance of increased glomerular pressure consequent to decreased preglomerular vascular resistance. Such a mechanism is in accord with many previous observations on autoregulatory behavior of the whole kidney $(6,7)$. This thesis has been questioned by Blantz, Israelit, Rector, and Seldin (17) who demonstrated that interruption of distal delivery by placement of an oil block in the proximal lumen does not lead to change in glomerular pressure as measured directly in rats with surface glomeruli. However, one might anticipate an increase of glomerular pressure only if the experimental kidney had retained its autoregulatory capability. Their data, as presented, provide no validation of autoregulatory behavior in their animals. Additionally, the magnitude of the increased glomerular pressure and consequently any measured differences between proximal and distal SNGFR might well be a function of the level of arterial pressure at which the measurements are made. Presumably, any proximal blockade-induced elevation of SNGFR might be greater at higher arterial pressures, thereby accentuating the differences between measurements of SNGFR from proximal and distal collection sites. Thus, the inability to describe a consistent difference between proximal and distal SNGFR in rats with a lower arterial pressure (about $110 \mathrm{~mm} \mathrm{Hg}$ ) is not necessarily at variance with the present observations $(16,28)$. Even the differences that were observed by Schnermann and colleagues $(11,20)$ in the rat were only about $7-10 \mathrm{nl} / \mathrm{min}$. In our own experiments in dogs, the difference between proximal and distal SNGFR was relatively modest at the lower arterial pressures. This apparent relationship between the level of arterial blood pressure and the magnitude of blockade-induced elevation of SNGFR may contribute to our understanding of the nature of the nonlinear relationship between glomerular hydrostatic pressure and the rate of perfusion through the loop of Henle $(13,14)$. At arterial pressures near the lower limits of the autoregulatory range, the reserve vasodilatory capability of the arterioles might be minimal; therefore, no more than a slight increase in glomerular pressure would be expected to occur at low rates of distal perfusion (14) or during complete luminal blockade (17).

The observation that higher control blood pressures 
are associated with much higher values for proximal SNGFR raises a question as to the true value for SNGFR in the dog under normal steady-state conditions (31). In view of the possibility that total fluid collection from a proximal tubule may serve to alter a distal feedback signal and thereby lead to an elevation of SNGFR, our own observations suggest that the true SNGFR may be reflected most accurately by measurements derived from distal collections. Several other recent studies also appear to support this conclusion. For example, in a recent preliminary publication, Hanssen's technique was applied to the dog by Bruns, Riley, Alexander, and Levinsky (32) and estimates of SNGFR were derived for both superficial and deep nephrons. It is interesting to note that the reported value for superficial SNGFR in hydropenic dogs averaged $63 \pm 5 \mathrm{nl} / \mathrm{min}$, a figure about $30-40 \%$ lower than that previously reported from the same laboratory when SNGFR was measured directly via micropuncture at proximal sites (29). Also, the lower. values derived from the use of Hanssen's technique resemble our own estimates of distal SNGFR much more closely, and they are substantially lower than the present values that were based on proximal collections.

By utilizing the data for whole kidney filtration fraction and SNGFR from the two collection sites, it is possible to calculate glomerular plasma flow. Such an approach may be justified in the dog since it has been shown that the filtration fraction for superficial nephrons is similar to that for the whole kidney (33). In the present study, the whole kidney filtration fraction averaged 0.33 and the distal SNGFR averaged $47 \mathrm{nl} / \mathrm{min}$. Using these data, average glomerular plasma flow was found to equal $142 \mathrm{nl} / \mathrm{min}$. This value falls within $10 \%$ of that obtained by Wallin, Rector, and Seldin (34) using the glomerular basement membrane antibody technique for measurements of regional plasma flow within the kidney. The close agreement can be obtained only when distal measurements of SNGFR are utilized. When calculations of glomerular plasma flow are based on proximal SNGFR measurements, values are obtained that exceed those reported by Wallin et al. by $50-60 \%$.

Finally, an estimate of average SNGFR for the entire kidney can be approached by an assessment of the relationship between whole kidney GFR and estimates of the population density of individual glomeruli. While the reported values for the number of glomeruli in dog kidneys vary substantially, the values reported by Oliver (35) may provide a reasonably conservative estimate; the use of Oliver's figures is justified further by the fact that the average kidney weight in his studies approximated that in the present experiments quite closely. Therefore, assuming a glomerular count of 12,650 glomeruli/g and an average GFR of $0.69 \mathrm{ml} / \mathrm{min} \cdot \mathrm{g}$, a mean value for SNGFR of $55 \mathrm{nl} / \mathrm{min}$ can be obtained. The discrepancy between this figure $(55 \mathrm{nl} / \mathrm{min})$ and that derived from distal collections $(47 \mathrm{nl} / \mathrm{min})$ is slight but may well be attributed to the possibility that the SNGFR of superficial nephrons is lower than that exhibited by deeper nephrons $(32,36)$. Orlowski and Bricker (37) performed glomerular counts and clearance measurements in the same dogs. Their value of $49.7 \mathrm{nl} / \mathrm{min}$ for average SNGFR agrees very well with the calculations. Thus, on balance, indirect calculations such as these support the concept that a quantitative estimate of superficial SNGFR in the dog is perhaps best obtained from distal collections of tubular fluid.

It is important to emphasize that the magnitude of any over-estimation of SNGFR that may result from the use of proximal collections may be influenced in large part by the coexistent degree of residual autoregulatory capability in a given experimental circumstance. As discussed previously (Fig. 5), the degree of overestimation may also depend, at least in part, on the level of arterial blood pressure at which the measurements are performed. At lower blood pressures which approach the lower limits of the autoregulatory range, little residual capacity for vasodilatation may remain and only a slight or negligible overestimation of true SNGFR would result. In addition, there are many conditions in which whole kidney autoregulatory behavior can be abolished and it is conceivable that its abolition may be accompanied by the loss of autoregulatory responsiveness on the part of individual nephrons as well. Under such conditions, an interruption of distal delivery might not elicit the appearance of an appropriate feedback signal; consequently, neither glomerular pressure nor SNGFR would rise. For these reasons, the present results do not imply that previous measurements of SNGFR that are based on proximal collections are necessarily invalid. However, the potential sources of error outlined above must be considered carefully in any quantitative interpretation involving changes in SNGFR and volume delivery in various experimental settings.

\section{ACKNOWLEDGMENTS}

The authors are grateful to J. T. Adkinson, G. S. Sides, L. Poe, R. Keith, M. Jackson, and M. Poole for their excellent technical assistance.

This study was supported by USPHS grants AM 10844, HL 11820, and HL 5848.

\section{REFERENCES}

1. Selkurt, E. E. 1963. The renal circulation. Handb. Physiol. 2(Sect. 2) : 1457.

2. Thurau, K. 1966. Nature of autoregulation of renal blood flow. Proc 3rd Int. Congr. Nephrol. 1: 162-173.

3. Shipley, R. E., and R. S. Study. 1951. Changes in renal blood flow, extraction of inulin, glomerular filtration rate, tissue pressure, and urine flow with acute altera- 
tions of renal artery blood pressure. Am. J. Physiol. $167: 676$.

4. Navar, L. G., and P. G. Baer. 1970. Renal autoregulatory and glomerular filtration responses to gradated ureteral obstruction. Nephron. 7: 301.

5. Navar, L. G., P. G. Baer, S. L. Wallace, and J. K. McDaniel. 1971. Reduced intrarenal resistance and autoregulatory capability after hyperoncotic loading. $\mathrm{Am}$. J. Physiol. $221: 329$.

6. Thurau, K., and E. Wober. 1962. Zur Lokalisation der autoregulativen Widerstandsänderungen in der Niere. Arch. Gesamte Physiol. Menschen Tiere (Pfluegers). 274: 553.

7. Navar, L. G. Minimal preglomerular resistance and calculation of normal glomerular pressure. Am. J. Physiol. 219: 1658, 1970.

8. Robertson, C. R., W. M. Dean, J. L. Troy, and B. M. Brenner. 1972. Dynamics of glomerular ultrafiltration in the rat. III. Hemodynamics and autoregulation. Am. J. Physiol. 223 : 1191.

9. Guyton, A. C., J. B. Langston, and G. Navar. 1964. Theory for renal autoregulation by feedback at the juxtaglomerular apparatus. Circ. Res. 15 Suppl. 1. 187.

10. Thurau, K., and J. Schnermann. 1965. Die Natriumkonzentration an den Macula densa-Zellen als regulierender Faktor für das glomerulum filtrat (Mikropunktionsversuche). Klin. Wochenschr. 43: 410.

11. Schnermann, J., J. M. Davis, P. Wunderlich, D. Z. Levine, and M. Horster. 1971. Technical problems in the micropuncture determination of nephron filtration rate and their functional implications. Pfluegers Arch. Eur. J. Physiol. $329: 307$.

12. Schnermann, J., F. S. Wright, J. M. Davis, W. v. Stackelberg, and G. Grill. 1970. Regulation of superficial nephron filtration rate by tubulo-glomerular feedback. Pfluegers Arch. Eur. J. Physiol. 318: 147.

13. Hierholzer, K., M. Butz, R. Muller-Suur, and I. Lichtenstein. 1972. Pressure measurements in proximal surface tubules of the rat-Single nephron filtration rate and tubuloglomerular feedback. Yale J. Biol. Med. $45: 224$.

14. Schnermann, J., A. E. G. Persson, and B. Ågerup. 1973. Tubuloglomerular feedback: nonlinear relation between glomerular hydrostatic pressure and loop of Henle perfusion. J. Clin. Invest. 52: 862.

15. Morgan, T. 1971. A microperfusion study of influence of macula densa on glomerular filtration rate. $\mathrm{Am}$. J. Physiol. 220: 186.

16. Bartoli, E., and L. E. Earley. 1973. Measurements of nephron filtration rate in the rat with and without occlusion of the proximal tubule. Kid. Int. 3: 372 .

17. Blantz, R. C., A. H. Israelit, F. C. Rector, Jr., and D. W. Seldin. 1972. Relation of distal tubular $\mathrm{NaCl}$ delivery and glomerular hydrostatic pressure. Kid. Int. 2: 22.

18. Clapp, J. R., G. A. Nottebohm, and R. R. Robinson. 1971. Proximal site of action of ethacrynic acid: Importance of filtration rate. Am. J. Physiol. 220: 1355.

19. Navar, L. G., A. C. Guyton, and J. B. Langston. 1966. Effect of alterations in plasma osmolality on renal blood flow autoregulation. Am. J. Physiol. 211: 1387.

20. Davis, J. M., J. Schnermann, and M. Horster. 1972. Micropuncture method for the determination of nephron filtration rate. A recollection study. Pfluegers Arch. Eur. J. Physiol. 333 : 271.
21. Wright, F. S., and G. Giebisch. 1972. Glomerular filtration in single nephrons. Kid. Int. 1: 201.

22. Vurek, G. G., and S. E. Pegram. 1966. Fluorometric method for the determination of nanogram quantities of inulin. Anal. Biochem. 16: 409.

23. Fuhr, J., J. Kaczmarczk, and C. D. Kruttgen. 1955. Eine einfache colorimetrische Methode zur Inulinbestimmung für Nieren-clearance-Untersuchungen bei Stoff wechselgesunden und Diabetikern. Klin. Wochenschr. $33: 729$.

24. Smith, H. W. 1956. Principles of Renal Physiology. Oxford University Press, New York. 212.

25. Steel, R. G. D., and J. H. Torrie. 1960. Principles and procedures of statistics. McGraw-Hill Book Company, New York. pp. 67 and 161.

26. Navar, L. G., J. B. Uther, and P. G. Baer. 1971. Pressure diuresis in dogs with diabetes insipidus. Nephron. 8: 97.

27. Liebau, G., D. Z. Levine, and K. Thurau. 1968. Micropuncture studies on the dog kidney. I. The response of the proximal tubule to changes in systemic blood pressure within and below the autoregulatory range. Arch. Gesamte Physiol. Menschen Tiere (Pfluegers). $304: 57$.

23. Landwehr, D. M., J. Schnermann, R. M. Klose, and G. Giebisch. 1968. Effect of reduction in filtration rate on renal tubular sodium and water reabsorption. $\mathrm{Am}$. J. Physiol. 215: 687.

29. Auld, R. B., E. A. Alexander, and N. G. Levinsky. 1969. Proximal tubular function during saline infusion and arterial clamping in the dog. Abstr. Am. Soc. Nephrol. 3: 3 .

30. Thurau, K., J. Schnermann, W. Nagel, M. Horster, and M. Wahl. 1967. Composition of tubular fluid in the macula densa segment as a factor regulating the function of the juxtaglomerular apparatus. Circ. Res. Suppl. II. 20 and 21: 79.

31. Schneider, E. G., R. E. Lynch, L. R. Willis, and F. G. Knox. 1972. Single-nephron filtration rate in the dog. Am. J. Physiol. 222: 667.

32. Bruns, F. J., A. L. Riley, E. A. Alexander, and N. G. Levinsky. 1972. The quantitative estimation of juxtamedullary (JM) and superficial (S) nephron filtration (SNFR) in the dog: control and saline loading. Clin. Res. $20(3): 588$.

33. Knox, F. G., L. R. Willis, J. W. Strandhoy, E. G. Schneider, L. G Navar, and C. E. Ott. 1972. Role of peritubule Starling forces in proximal reabsorption following albumin infusion. Am. J. Physiol. 223: 741.

34. Wallin, J. D., F. C. Rector, Jr., and D. W. Seldin. 1971. Measurement of intrarenal plasma flow with antiglomerular basement-membrane antibody. Am. J. Physiol. 221 : 1621.

35. Oliver, J. 1968. Nephrons and Kidneys. Harper and Row, Publishers, New York. 48.

36. Bonvalet, J. P., P. Bencsath, and C. De Rouffignac. 1972. Glomerular filtration rate of superficial and deep nephrons during aortic constriction. Am. J. Physiol. 222: 599

37. Orlowski, T., and N. S. Bricker. 1964. The relationship between renal function and the direct glomerular count in normal dogs. Acta Med. Pol. 5 : 247. 